\title{
475850 - SEIZURE IN A CANCER PATIENT ON METHADONE
}

\author{
Jafer Ali, MD, Don Woods, MD \\ Anesthesiology/Pain Medicine, University Hospitals of Cleveland/Case Medical \\ Center, Cleveland, OH, USA
}

Purpose: Methadone is a long acting opioid that is often used effectively to treat cancer pain. Like all opioids, it has risks and side effects. This is a case report of a patient with metastatic rectal cancer who was on large doses of methadone and had a seizure.

Clinical Features: A middle-aged woman with stage IV metastatic rectal cancer (diaphragm, liver, lungs) was admitted directly to the hospital from her oncologist's office secondary to uncontrolled pain. The patient was not a candidate for any additional surgery nor radiation/chemotherapy. Her home regimen of methadone $60 \mathrm{mg}$ po tid and methadone $30 \mathrm{mg}$ po q 3hours prn was ineffective in controlling her pain. Over a 3 day hospitalization, the primary service increased her methadone to $90 \mathrm{mg}$ po tid and methadone 90 mg po q 2 hours prn. She was usually maximizing her prn methadone, yielding a daily po methadone intake close to $1300 \mathrm{mg}$ per day. On hospital day \#4 the patient was discharged to home on this regimen. The following day she lost consciousness for several minutes and appeared to have a seizure. She went to her local ER where a seizure secondary to methadone was suspected. The patient saw her oncologist 2 days later and was seen in the pain clinic the same day. She was placed on the fentanyl patch $100 \mathrm{mcg} / \mathrm{hr}$, oxycodone $10 \mathrm{mg}$ qid, and ibuprofen $800 \mathrm{mg}$ q 6 hours prn. The methadone was reduced to $60 \mathrm{mg}$ po bid. During a phone follow-up one week later the patient reported her pain was well controlled on this regimen and denied any additional seizures. Future placement of a tunneled epidural catheter was discussed, but deferred secondary to adequate pain relief. The patient died 6 weeks after her initial pain clinic visit. The patient did consent to this being submitted as a case report.

Conclusion: Seizure is a known side effect of methadone therapy. This case report describes a seizure that occurred in a cancer patient that was on large dose methadone therapy that had been rapidly increased. It also demonstrates the benefits of opioid rotation and multimodal therapy for patients with cancer pain.

References: Morgan, Edward and Maged Mikhail. Clinical Anesthesiology. New York: McGraw-Hill, 2006 